doubt that an increase in diagnostic accuracy is possible by using both methods together. Mammography is capable of demonstrating an impalpable lesion. Even with the most careful technique, however, mammography will still miss a significant and irreducible number of lesions and must not be used as a substitute for clinical examination. In our experience mammography is likely to miss carcinoma in the young dense breast or in the bulky invasive type of lesion, poorly differentiated from the surrounding tissues and showing no secondary signs such as calcification or skin-thickening. Such a lesion is usually clinically obvious.

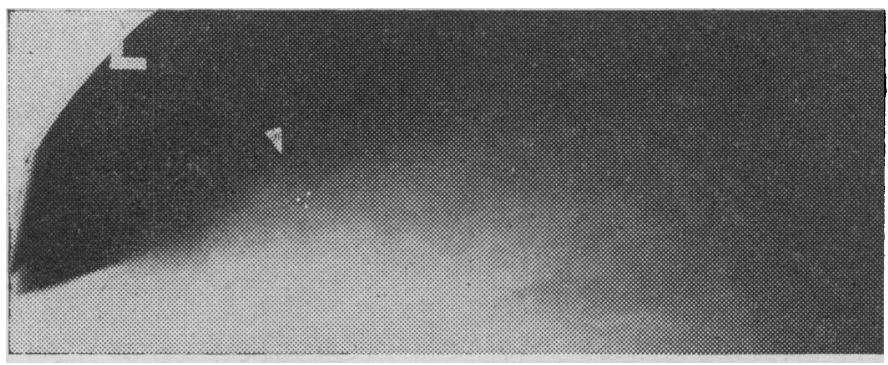

FIG. 4.-Clinically-oval lump in breast, probably fibroadenoma. Mammogram-well-defined mass 6 by $3 \mathrm{~cm}$. with $1 \mathrm{~cm}$. irregular density showing tiny calcifications at lateral aspect-benign lesion + carcinoma. Histology-large area fibroadenosis with $1 \cdot 5-\mathrm{cm}$. scirrhous carcinoma.

In the palpable lesion mammography may allow a positive diagnosis of carcinoma to be made preoperatively or, conversely, may point to a benign lesion. Thus in some of the carcinomas considered to be equivocal by the surgeon the evidence of the mammogram permitted mastectomy to be carried out without resort to preliminary biopsy. Less frequently, in a benign lesion considered to be carcinoma or equivocal by the surgeon the mammogram may indicate the need for biopsy rather than mastectomy. It cannot be stressed too strongly that mammography does not as yet replace the need for biopsy, and this is particularly true in the younger age groups where mammography is less accurate (Copland and Scott, 1968). Even now a confident radiographic diagnosis of carcinoma may support the clinical diagnosis sufficiently to obviate biopsy in elderly or infirm patients in whom the primary treatment is to be by palliative radiotherapy, hormones, or cytotoxic drugs. Mammography may also be helpful in a patient with known or suspected benign disease where a carcinoma may be demonstrated within an area of fibrocystic disease (see Fig. 4). The mammogram can also direct the pathologist's attention to the relevanto area of the surgical specimen in such cases.

Thomas and McDonald (1967) sounded a cautionary note $\overrightarrow{\bar{F}}$ on the accuracy of mammography. They reported only ao $61 \%$ true positive rate in the detection of proved carcinoma, and they also found that Egan has not maintained his initialo high accuracy. The majority of their patients, however, seem to have been in the younger age groups, and there is no doubt that accuracy in mammography increases with the age of the $e^{\infty}$ patient. It is possible that discrepancies in accuracy between $\overrightarrow{0}$ reports reflect the varying incidence of patients in younger age groups.

Further improvements in technique will increase the value of mammography, and research on such improvements must be stimulated and supported (Fitts, 1966). Mammographyir must be used only as a complement to clinical examination ando not as a substitute, otherwise the method will be brought into+ disrepute.

\section{REFERENCES}

Bohrer, S. P. (1964). British fournal of Radiology, 37, 237. Copland, M. M., and Scott, W. G. (1968). American fournal of Surgery, 116, 57

Egan, R. L. (1960). Radiology, 75, 894.

Egan, R. L. (1964). Mammography. Springfield, Illinois, Thomas.

Fitts, W. T. (1966). Surgery, Gynecology and Obstetrics, 122, 1077. Ф

Ingleby, H., and Gershon-Cohen, J. (1960). Comparative Anatomy, $\rightarrow$ Pathology and Roentgenology of the Breast. Philadelphia, University 8 S
of Pennsylvania Press.

Irvine, R. W., and James, W. B. (1969). In press.

Leborgne, R. A. (1953). The Breast in Roentgen Diagnosis. Montevideo,o Impresora Uruguya.

Salomon, A. (1913). Archiv für klinische Chirurgie, 101, 573.

Samuel, E., and Young, B. (1964). Clinical Surgery, edited by C. Rob and R. Smith, p. 431. London, Butterworths.

Thomas, M. A., and McDonald, E. J. (1967). Medical Annals of the District of Columbia, 36, 468 .

Young, G. B. (1968). Fournal of the Royal College of Surgeons of $\frac{0}{3}$ Edinburgh, 13, 12 .

\title{
Percutaneous Needle Nephrostomy
}

C. S. OGG,* M.D., B.SC., M.R.C.P.; H. M. SAXTON, $\dagger$ M.R.C.P., F.F.R.; J. S. CAMERON, ${ }^{*}$ M.D., B.SC., M.R.C.P.

Gummary : Percutaneous nephrostomy is a simple tech$\checkmark$ nique for temporary drainage of an obstructed kidney. Under local anaesthesia a ureteric catheter is passed through a Vim-Silverman needle into the renal pelvis and is connected to a drainage bag. Seven cases (six successful) in which this method was used are described and the indications are discussed. It has been free from complications, is acceptable to patients, and is felt to represent a useful addition to the methods available for the treatment of obstruction of the upper urinary tract.

* Renal Physician, Guy's Hospital, London S.E.1.

† Radiologist, Guy's Hospital, London S.E.1.

\section{Introduction}

In the management of obstruction of the upper urinary tract it is sometimes desirable to provide temporary relief of the obstruction, usually until definitive treatment can be under- $\frac{\mathrm{D}}{\mathbb{D}}$ taken. Retrograde catheterization and operative nephrostomy $\stackrel{\rho}{\stackrel{\rho}{\odot}}$ are the measures commonly employed for this purpose, but $\varrho$ each has disadvantages. It may be impossible to pass a ureteric catheter above the obstructing lesion; even when passed it is 8 uncomfortable and often becomes dislodged downward into the bladder. On the other hand, the surgeon may hesitate to undertake nephrostomy because it involves a relatively major procedure requiring a general anaesthetic for what may be a transitory obstruction. Percutaneous nephrostomy is a simple 
and effective alternative without these drawbacks, and in this paper we describe our experience with the method.

\section{Technique}

The procedure was first described by Goodwin, Casey, and Woolf (1955) and has recently been modified by Cobb (1967). We have based our technique on the latter author's description but have used fluoroscopy with image intensification for the initial localization of the kidney. The procedure may be described as having two stages-percutaneous pyelography, followed by percutaneous nephrostomy. The kidney is first made visible for fluoroscopy, commonly by high-dose urography. In most cases the faint outlines of the calices and pelvis have become visible, but occasionally we have been guided by the nephrogram alone. When the degree of opacity seems adequate the patient is turned prone and settled as comfortably as possible. A fine exploring needle-for example, Harris lumbar puncture No. 5-is introduced under local anaesthesia into the pelvi-caliceal system. With acute obstruction the urine is under pressure and wells up when the needle enters the pelvis, but in one of our cases where the obstruction was of long standing it was necessary to aspirate to confirm the correct position of the needle.

Contrast medium-for example, 10-15 ml. of Conray 280is injected via a flexible connexion to produce better visualization of the upper urinary tract and to confirm the presence and site of obstruction. The needle is then withdrawn, noting the depth to which it has been introduced. Next a VimSilverman renal biopsy needle is passed, under fluoroscopic control, to a corresponding depth and so into the renal pelvis. The stylet is removed, and, provided urine emerges, a No. 5 (French) whistle-tipped ureteric catheter is threaded through the needle and advanced well into the pelvis. Usually the catheter passes easily into the pelvis, but occasionally rotation of the bevel or suitable angulation of the needle is required. The needle is withdrawn over the catheter, which is then fixed to the skin and connected to a drainage bag. Drainage continues until the obstruction has been relieved, and the catheter is then removed.

\section{Case 1}

This patient was a 52-year-old woman who in 1966 was found to have lymphosarcoma. She had been treated with cyclophosphamide and remained reasonably well until February 1968. She then complained of haematuria and left renal colic, and shortly afterwards became anuric. Bilateral retrograde pyelography showed that both ureters were severely narrowed in their mid-portions. At right nephrostomy extensive retroperitoneal deposits of lymphosarcoma were found. The nephrostomy did not drain adequately and the blood urea rose to $280 \mathrm{mg}$. $/ 100 \mathrm{ml}$. On 1 March 1968 a percutaneous pyelogram (Fig. 1) showed complete obstruction of the left ureter. Left percutaneous nephrostomy was performed and good drainage resulted. Three days later the blood urea had fallen to $38 \mathrm{mg} . / 100 \mathrm{ml}$. Radiotherapy to the abdomen was begun on $8 \mathrm{March}$, and the course was completed on 3 April. Spontaneous passage of urine began 10 days after the beginning of treatment, and injection of contrast medium through the percutaneous catheter showed that the ureter was no longer obstructed (Fig. 2). On 23 March the nephrostomy catheter was removed. At first the patient appeared to be maintaining her improvement, but further lymphosarcomatous masses developed and six weeks later she died.

\section{Case 2}

A 61-year-old man underwent abdomino-perineal resection for carcinoma of the rectum in 1967. In October 1968 he was admitted to hospital in a semicomatose state with a history of nine days' anuria and with a blood urea of $310 \mathrm{mg} . / 100 \mathrm{ml}$. Peritoneal dialysis produced a gradual improvement, and retrograde ureterograms showed bilateral obstruction at the pelvic brim. Percutaneous nephrostomy on 15 October produced adequate drainage of the left kidney, and his blood urea stabilized at $200 \mathrm{mg} . / 100 \mathrm{ml}$. Ten days later ureterostomy was performed and the ureteric catheter was removed. Nine months later he was well, with a blood urea of about $120 \mathrm{mg} . / 100 \mathrm{ml}$.

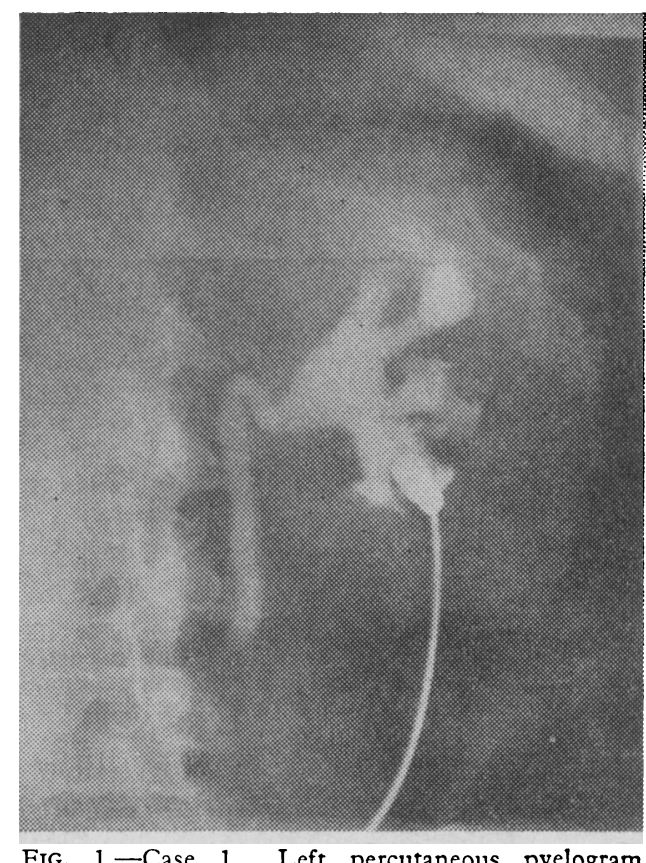

FIG. 1.-Case 1. Left percutaneous pyelogram L3-4 level.

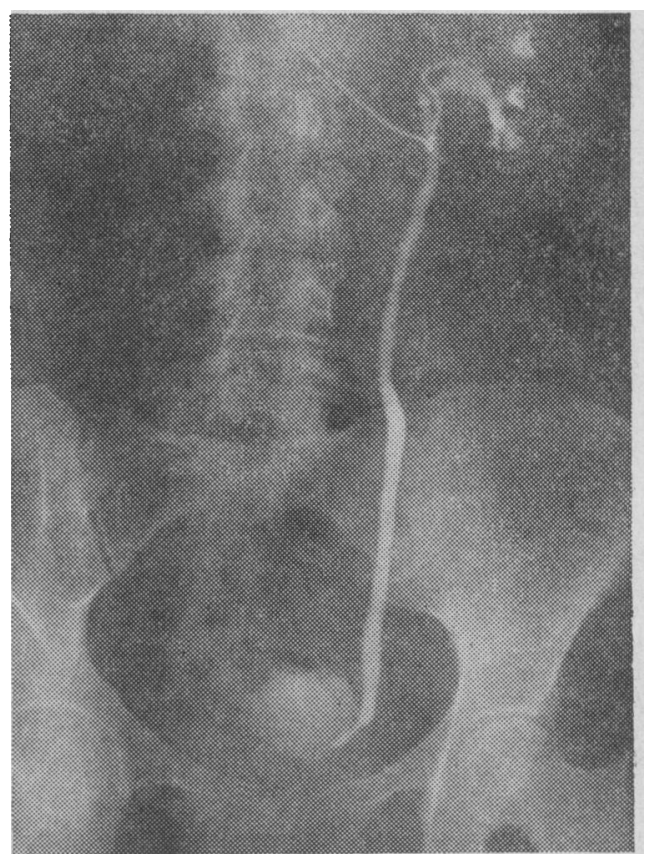

Fig. 2.-Case 1. Injection of contrast medium through the percutaneous catheter shows that after radiotherapy the ureter is patent throughout its length (22 March 1968).

\section{Case 3}

This 47-year-old man with epilepsy had been treated with phenobarbitone and phenytoin for several years. Three weeks before admission he was put on acetazolamide, and 17 days later developed bilateral loin pain and became anuric. On admission his blood urea was $118 \mathrm{mg} . / 100 \mathrm{ml}$., but later rose to $212 \mathrm{mg} . / 100 \mathrm{ml}$. At cystoscopy debris was seen extruding from the left ureteric orifice 
and it was impossible to catheterize either ureter. Urography confirmed the presence of bilateral obstruction, and on 27 February 1969 percutaneous nephrostomy was performed on the left side. This drained well, and soon afterwards the right side cleared spontaneously, possibly owing to the osmotic diuresis produced by the urographic contrast. The blood urea rapidly returned to normal levels and an antegrade pyelogram showed that the left ureter was patent. After seven days the catheter was removed. An intravenous urogram was normal and he was discharged from hospital. It was presumed that the acetazolamide had produced ureteric "sludge" with bilateral obstruction.

\section{Case 4}

A 21-year-old man with a long history of enuresis was admitted to hospital because of sudden onset of left loin pain. He had a urinary infection and a blood urea of $188 \mathrm{mg} . / 100 \mathrm{ml}$. At urography both kidneys were found to be large, with minimal function on the left side and no excretion on the right. Voiding cystography showed a grossly trabeculated bladder with no outlet obstruction and no reflux, the appearances suggesting a neurogenic bladder. Left retrograde pyelography showed a large hydronephrosis with pelvi-ureteric narrowing and angulation. Because of the risk of infection following retrograde pyelography a percutaneous nephrostomy was performed on 5 May 1969. When the pelvis was punctured it was found that the urine was not under pressure, and aspiration was needed to confirm the correct position of the needle. The low pressure was attributed to the chronicity of the obstruction. Drainage was continued until pyeloplasty three days later. His right kidney was later removed. At the time of writing his blood urea was steady at about $150 \mathrm{mg} . / 100 \mathrm{ml}$.

\section{Case 5}

This 68-year-old man underwent abdomino-perineal resection of a rectal carcinoma in February 1969. In April he noticed diminished urine output and he became anuric on 1 May. Six days later he was admitted to Guy's Hospital with a blood urea of $280 \mathrm{mg} . / 100$ $\mathrm{ml}$. High-dose urography confirmed the presence of obstruction on both sides, and bilateral percutaneous nephrostomy was performed. In spite of some blood-staining both catheters drained well, and within two days his blood urea had fallen to $32 \mathrm{mg} . / 100 \mathrm{ml}$. This fall was accompanied by a massive diuresis, reaching a peak of 10 litres in one day. Subsequent laparotomy showed extensive peritoneal deposits; his condition deteriorated and he died on 2 June.

\section{Case 6}

This 68-year-old man was known to have a solitary left kidney with moderate pelvi-ureteric narrowing and hydronephrosis. In May 1969 he became oliguric, urography showing a considerable increase in the hydronephrosis. His blood urea was $250 \mathrm{mg} . / 100 \mathrm{ml}$. In spite of clear visualization of the kidney it proved impossible to puncture the pelvis even when a relatively long needle was used. Subsequently a nephrostomy was performed and later a pyeloplasty ; no indication of trauma due to the needling was seen.

\section{Case 7}

This 40-year-old man had a left pyelolithotomy in 1967. A right-sided stone present at that time increased in size, and in March 1969 a right pyelolithotomy was carried out. This was a difficult operation and was followed by severe urinary infection. Two months later urography showed no excretion by the right kidney, and retrograde pyelography revealed obstruction at the pelvi-ureteric junction. It was decided to undertake percutaneous nephrostomy to determine the extent of any residual function. This was performed on 23 May and a good flow of urine was obtained. A week later the creatinine clearance of the right kidney was $11.3 \mathrm{ml}$. per minute, while that of the left side was $42 \mathrm{ml}$. per minute. It therefore appeared that the right kidney provided a significant proportion of the total renal function, and a right ureterocalicotomy was successfully carried out on 10 June.

\section{Discussion}

The practical value of a technique which affords reliable temporary relief of obstruction will be readily apparent. Time is gained during which the patient's condition can improve, infection be controlled or prevented, and the diagnosis be confirmed or treatment planned. Though the indications are $\underline{T}$ admittedly restricted, it is difficult to see why the procedure 3 has not been more widely used. Renal puncture is generally $\mathbb{D}$ accepted as safe, whether for renal biopsy or for the diagnostic $c$. tapping of cysts. Percutaneous pyelography, an essential pro- $\Rightarrow$ liminary to this method, has been advocated in a number of $\stackrel{5}{+}$ papers over the past 15 years (Weens and Florence, 1954 ; 을 Wickbom, 1954 ; Casey and Goodwin, 1955 ; Floyd and Guy, 음 1956 ; Lundin and Wadström, 1965; Maldonado, 1956 ; $\frac{\bar{\omega}}{7}$ Bosniak, Scheff, and Kaufman, 1968 ; Lalli, 1968). Yet once $\mathbb{\complement}$ the renal pelvis has been located and visualized in this way the additional manœuvre required for drainage is so simple $\vec{\bullet}$ that we can see little object in performing antegrade pyelography for obstruction without at the same time attempting $\vec{\omega}$ percutaneous nephrostomy.

Our experience of the method has been that in six of the $\frac{0}{3}$ seven cases in which it was attempted it was straightforward, and in all cases it was without complications. In one patient $i$ (Case 6), though the pelvis was considerably enlarged and $\infty$ clearly visible, it was not possible to puncture it. At the time of it was thought that this was due to inspissation of the pelvic $f$ contents, but at subsequent nephrostomy that did not prove $ᄋ$ to be the case, and it was not possible to see why puncture had failed. In retrospect it is thought that it might have been $\vec{\omega}$ helpful to establish the relative depths of the needle and kidney by taking a single $x$-ray film in the transverse plane.

So few accounts have been given of this technique that it would be unrealistic to discuss the incidence of complications. $\stackrel{\mathbb{D}}{-}$ In the two patients (Cases 4 and 7) who have undergone $\vec{\bullet}$ exploration of the kidney shortly after removal of the catheter 8 there was no indication of any harmful effect on the kidney or pelvis. As Goodwin et al. (1955) and Cobb (1967) point 0 out, there are no vital structures in the path of the needle except the renal vessels. The probability of accidental puncture $\bar{o}$ of a vessel during exploratory needling under screen control is

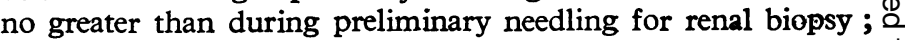
such accidental puncture is most unlikely to cause significant $\overrightarrow{\vec{O}}$ bleeding. Once the pelvi-caliceal system has been visualized 3 by direct injection of contrast medium it becomes possible to direct the larger (Vim-Silverman) needle to the lateral side of the pelvis, so avoiding the larger renal vessels.

Obstruction of the catheter may occur, and Cobb (1967) successfully inserted a second catheter when this happened. A possible cause of such obstruction is kinking due to excessive 3 coiling in the renal pelvis, but though several of our cases $\delta$ have shown marked coiling this has not affected drainage, $₹$ possibly because we have usually employed "woven" catheters 0 as Cobb recommended. The surprising feature of the technique ${ }_{N}$ is the good drainage produced by the relatively narrow catheter or - so much so that in Case 3, where the output through two $\frac{7}{0}$ catheters reached as high as 10 litres in a day, there was some difficulty in maintaining adequate fluid balance. The period $N$ of drainage in this series ranges from 3 to 29 days, but Case $3 \mathrm{~N}$ of Goodwin et al. drained satisfactorily for five months and 0 the tube showed no sign of encrustation. Though we have not used the method in the presence of infection, the experience of Goodwin et al. (1955) and of Cobb (1967) shows that this $\stackrel{\oplus}{+}$ is not a bar to the technique. In their cases the infection 0 responded rapidly to adequate drainage.

Cases in which percutaneous nephrostomy is suitable for $\stackrel{\mathbb{D}}{\mathscr{Q}}$ use do not often occur. The indications for the procedure $\stackrel{\mathbb{Q}}{\varrho}$ may widen as it becomes more generally adopted, but at present $\bar{\sigma}$ they may be summarized as follows:

(1) Short-term relief of obstruction in the anuric patient in whom retrograde catheterization is impossible. This may be because of technical difficulty with catheterization, because of the severity of the ureteric narrowing, because the condition of the patient precludes anaesthesia, or because the ureter is not available for catheterization-for example, after implantation into an ileal loop. 
(2) Short-term relief of obstruction in a patient who is awaiting pyeloplasty, when there is a risk that pyonephrosis may developfor example, after retrograde catheterization. We would also suggest that it might be of value to produce preliminary decompression of the renal pelvis and allow contraction of the stretched tissues, making subsequent surgery easier.

(3) Assessment of potential recovery of an obstructed kidney or in a non-functioning moiety of an obstructed duplex kidney.

For the most part these indications require little comment. Cases with suspected malignant disease involving both ureters raise difficult problems of management, since the benefit resulting from relief of obstruction is likely to be temporary and it may at times be more humane not to allow the patient to recover sufficiently from renal failure to experience the direct effects of the growth. But in other cases of anuria due to obstruction there is every advantage in improving the general condition of the patient and diminishing the risk of infection before curative surgery. In unilateral obstructive disease there is an interesting further possibility, since a period of satisfactory drainage makes it possible to assess the likely return of function with adequate relief of obstruction (see our Case 7 and Goodwin et al. (1955) Case 2). This, in our view, offers a most promising area for further extension of the method. The importance of conserving renal tissue is undoubted, yet after a period of chronic obstruction it may be impossible to predict whether adequate function can be restored to a kidney even if the obstruction is satisfactorily relieved. Percutaneous nephrostomy offers a practical way of resolving such uncertainties.
The particular technique recommended is not definitive. For the purpose of simple drainage the No. 5 ureteric catheter is adequate, but narrower catheters could be preferable for other purposes-for example, long-term manometric studies and the instillation of antibiotics, chemotherapeutic agents, or stone-dissolving fluids. At times a wider catheter might be needed. Whatever the future extensions of the method, the existing technique is, in our view, a significant advance in the management of obstructive disease of the upper urinary tract.

We would like to thank the physicians and surgeons who referred these patients to us, and Mr. F. R. Kilpatrick and Mr. C. H. Kinder for their advice and help.

\section{REFERENCES}

Bosniak, M. A., Scheff, S., and Kaufman, S. (1968). Fournal of Urology, 99, 241.

Casey, W. C., and Goodwin, W. E. (1955). Fournal of Urology, 74, 164. Cobb, B. (1967). Fournal of Urology, 98, 309.

Floyd, E., and Guy, J. C. (1956). Fournal of the Medical Association of Georgia, 45, 13.

Goodwin, W. E., Casey, W. C., and Woolf, W. (1955). Fournal of the American Medical Association, 157, 891.

Lalli, A. F. (1968). Radiology, 90, 331.

Lundin, E., and Wadström, L. B. (1965). Acta Chirurgica Scandinavica. $130,267$.

Maldonado, J. (1966). Fournal of Urology, 96, 651.

Weens, H. S., and Florence, T. J. (1954). Fournal of Urology, 12, 589.

Wickbom, I. (1954). Acta Radiologica, 41, 505.

\title{
Latent Cytomegalovirus Infection in Blood Donors
}

\author{
PETER DIOSI,* M.D.; EVA MOLDOVAN,* M.D.; NICHOLAS TOMESCU, $†$ M.D.
}

\begin{abstract}
Summary : Twenty-one out of 32 apparently healthy blood donors aged 21 to 65 years yielded positive complement fixation tests with a cytomegalovirus antigen, at titres ranging from $1: 8$ to $1: 64$. Virus was present in leucocyte cultures of fresh peripheral blood of two seropositive subjects from a total of 35 donors examined. Plasma and 48-hour stored blood specimens failed to disclose virus in culture. Viruria could not be demonstrated, and there was no evidence of recent illness in the study group. These findings suggest that subclinical viraemia is not uncommon in blood donors.
\end{abstract}

\section{Introduction}

Wyatt et al. (1951) were the first to assume that cytomegalovirus infection could be the result of the introduction of virus into a non-carrier by transfusion with blood from a carrier. Since then the occurrence of cytomegaloviraemia has been chown by the recovery of cytomegalovirus strains from the buffy-coat layer of a blood sample received from a patient with giant-cell type hepatitis (Stulberg et al., 1966), as well as from washed leucocytes obtained from the peripheral blood in cases of congenital cytomegalic inclusion disease (Demidova et al., 1968), post-transfusion mononucleosis (Foster and Jack, 1968),

* Institute of Hygiene, Timisoara, Rumania. t Centre of Blood Transfusions, Timisoara, Rumania. post-perfusion syndrome (Lang et al., 1968), lymphatic leukaemia (Harnden et al., 1967 ; Diosi and Roth, 1969), and myeloid leukaemia (Jack et al., 1968).

Since generalized cytomegalic inclusion disease and cytomegalovirus mononucleosis have been shown to develop after the transfusion of fresh blood (Kääriäinen et al., 1966a; Henson, 1967.), in connexion with renal haemodialysis (Cutforth et al., 1968), or surgery with extracorporeal circulation (Anderson and Larsson, 1963 ; Embil et al., 1968 ; Marton et al., 1968 ; Foster and Jack, 1969), attempts were made in the present study to recover cytomegalovirus from donated fresh blood and from blood specimens stored for 48 hours before their administration.

\section{Materials and Methods}

The study group, which came from the Blood Transfusion Centre in Timişoara, consisted of 35 blood donors aged 21 to 65 years, belonging at random to various blood groups. Routine haemograms and urine analyses yielded normal values in each case, while serology for syphilis and hepatocellular function tests were negative. None of the individuals surveyed showed any sign of disease.

\section{Virological Techniques}

Details of the laboratory methods employed have for the most part been reported elsewhere (Diosi and Roth, 1969). 